Bull. Mater. Sci., Vol. 30, No. 1, February 2007, pp. 69-71. (c) Indian Academy of Sciences.

\title{
Effect of transient change in strain rate on plastic flow behaviour of low carbon steel
}

\author{
A RAY, P BARAT*, P MUKHERJEE, A SARKAR and S K BANDYOPADHYAY \\ Variable Energy Cyclotron Centre, 1/AF, Bidhan Nagar, Kolkata 700 064, India
}

MS received 19 May 2006; revised 10 November 2006

\begin{abstract}
Plastic flow behaviour of low carbon steel has been studied at room temperature during tensile deformation by varying the initial strain rate of $3.3 \times 10^{-4} \mathrm{~s}^{-1}$ to a final strain rate ranging from $1.33 \times 10^{-3} \mathrm{~s}^{-1}$ to $2 \times 10^{-3} \mathrm{~s}^{-1}$ at a fixed engineering strain of $12 \%$. Haasen plot revealed that the mobile dislocation density remained almost invariant at the juncture where there was a sudden increase in stress with a change in strain rate and the plastic flow was solely dependent on the velocity of mobile dislocations. In that critical regime, the variation of stress with time was fitted with a Boltzmann type Sigmoid function. The increase in stress was found to increase with final strain rate and the time elapsed in attaining these stress values showed a decreasing trend. Both of these parameters saturated asymptotically at a higher final strain rate.
\end{abstract}

Keywords. Steels; stress-strain measurement; plastic flow; mechanical properties; metallurgy.

\section{Introduction}

The plasticity of metals and alloys is a consequence of the motion of line defects or dislocations, under the action of an applied stress (Hirth and Lothe 1982; Nabarro 1979-87; Suzuki et al 1991). Their motion is obstructed by point defects, impurities, electrons, phonons, other dislocations and the Peierls (1940) barrier that originates from the crystal structure itself. The stress-strain curve of the materials in the plastic regime is thus governed by the dynamics of dislocations. The plastic deformation of solids is often described by the equations based on the variables of deformation state of the form

$$
F(\sigma, \varepsilon, \dot{\varepsilon}, T)=0,
$$

where $\sigma$ is the flow stress, $\varepsilon$ the true strain, $\dot{\varepsilon}$ the true strain rate, and $T$ the temperature. Furthermore, one can express the true strain rate, $\dot{\varepsilon}$, as a function of $\sigma$ for fixed $\varepsilon$ and $T$. The flow stress, $\sigma$, is dependent on the grain size of the material. The effect of grain size, $D$, on $\sigma$ of metals is generally considered in terms of the Hall-Petch (Hall 1951; Petch 1953) relation:

$$
\sigma=\sigma_{i}+K_{\mathrm{HP}} D^{-1 / 2}
$$

where $\sigma_{i}$ is the lattice friction stress and $K_{\mathrm{HP}}$ the HallPetch parameter. In metals, the lattice friction stress, $\sigma_{i}$, is generally dependent on strain, $\varepsilon$, strain rate, $\dot{\varepsilon}$ and temperature, $T$, while $K_{\mathrm{HP}}$ is relatively independent of these parameters.

\footnotetext{
*Author for correspondence (pbarat@ veccal.ernet.in)
}

Several experimental techniques are adopted in order to study the plastic deformation. Some of these techniques include strain rate change test, stress change test and stress rate change test (Onodera 2002; Hayakawa et al 2003), all within a short interval of time. The aim of these deformation transient experiments is to study the deformation kinetics and the associated microstructural changes. In the present work, we have carried out strain rate change tests on low carbon steel and shown the effect of sudden change of strain rate on the plastic flow behaviour primarily governed by the velocity of mobile dislocations.

\section{Experimental}

The composition of the low carbon steel used in the present study was (in wt. \%): $0.15 \mathrm{C}, 0.33 \mathrm{Mn}, 0.04 \mathrm{P}, 0.05 \mathrm{~S}$, $0 \cdot 15 \mathrm{Si}$ and rest iron. The gauge length of the cylindrical tensile specimens was $25 \mathrm{~mm}$. The uniaxial tensile tests were performed at room temperature with the help of a servo controlled INSTRON (model 4482) machine. The initial crosshead speed was $0.5 \mathrm{~mm} / \mathrm{min}$ (corresponding to an initial engineering strain rate of value, $3.3 \times 10^{-4} \mathrm{~s}^{-1}$ ) for all the specimens up to an engineering strain of $12 \%$. The cross head speed was then changed to different values, i.e. $2,4,5,6,8,10,12,15,18,20,25$ and $30 \mathrm{~mm} / \mathrm{min}$. The tensile load and displacement were measured using a computer-controlled interface at a sampling rate of 20 points/s.

Figure 1 shows a segment of a typical true stress vs true strain curve in the plastic regime showing a sudden increase in true stress at $11.33 \%$ true strain (corresponding to $12 \%$ engineering strain) due to a sudden increase in the 
strain rate. The yield stress values for the specimens were found to lie within 460-480 MPa. Prior to strain hardening, extensive serrations were noticed in all the specimens. This oscillation of stress is associated with the heterogeneous deformation behaviour of the material, mainly due to the barrier-controlled dynamics of dislocations (Kok et al 2003). The present study was carried out in the plastic regime where the deformation was nearly homogeneous.

\section{Strain rate sensitivity analysis}

It is known that an increase in strain rate increases the tensile strength of metals (Mignogna et al 1970). The sudden change in the strain rate during tensile deformation could be a very useful approach to understanding the dynamics of dislocations. The strain rate sensitivity index, $m$, which is defined as

$$
m=\ln \left(\sigma_{2} / \sigma_{1}\right) \ln \left(\dot{\varepsilon}_{2} / \dot{\varepsilon}_{1}\right),
$$

where subscripts ' 1 ' and ' 2 ' stand for the initial and final values, respectively was calculated and the values were found to be $\sim 0.01$ for all the samples. A Haasen (1958) plot, $\Delta \sigma /(T \Delta \ln \dot{\varepsilon})$ vs $\sigma-\sigma_{\mathrm{Y}}$, which is commonly used to study the nature of dislocation interactions in monotonically deformed materials (Mulford 1979), obtained from the true stress-true strain data for all the samples is shown in figure 2. Here, $\Delta \sigma$ is the change of stress due to a change in the strain rate, $\dot{\varepsilon} ; \sigma-\sigma_{\mathrm{Y}}$ the effective flow stress, $\sigma_{\mathrm{Y}}$ the yield stress and $T$ the temperature.

Generally metals with a few regularly spaced dislocations at room temperature, exhibit linear Haasen plot passing through the origin. Its slope is proportional to the inverse of the operational activation area (Kocks et al 1975) and is expected to vary as the number of mobile

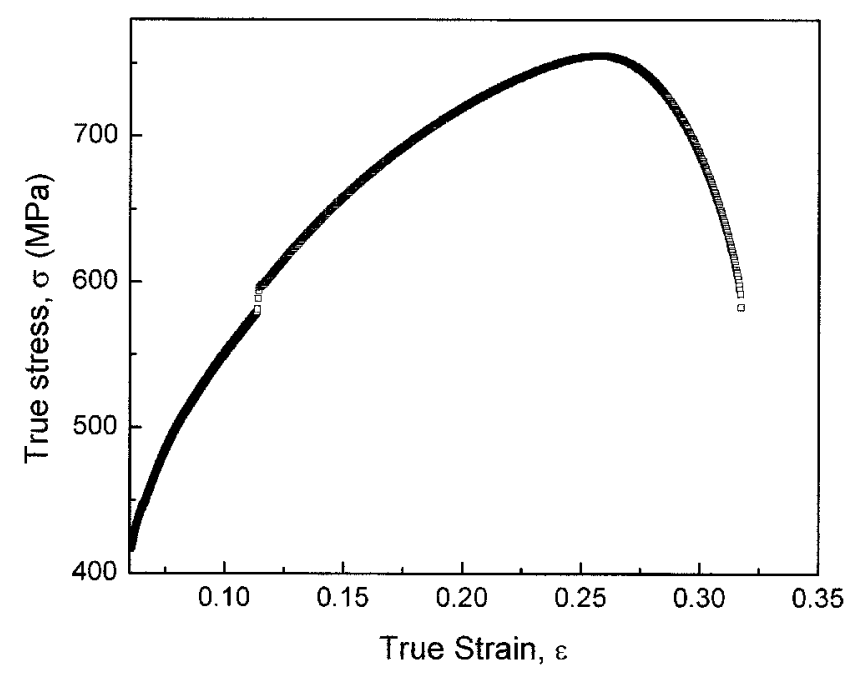

Figure 1. A segment of a typical true stress vs true strain curve in the plastic regime of the low carbon steel specimen for initial crosshead speed of $0.5 \mathrm{~mm} / \mathrm{min}$ and a final cross-head speed of $10 \mathrm{~mm} / \mathrm{min}$. dislocations changes with time (Kaschner and Gibeling 1996). However, since the strain rate sensitivity index, $m$, is proportional to the slope of the Haasen plot, the apparent constancy in the parameter, $m$, suggested that the mobile dislocation density did not change appreciably during the transient changes of strain rate (Mulford 1979).

Again, it is reported that (Reid et al 1965), the dislocation density, $\rho$, in metals evolves with strain, $\varepsilon$, by the following relation:

$$
\rho=\rho_{0}+M \varepsilon^{\beta},
$$

where $\rho_{0}$ is the initial dislocation density, $M$ the multiplication factor that does not depend either on the strain rate or temperature and $\beta$ the strain exponent, assumed to be unity in most cases. This relation justifies that the total dislocation density after a sudden increase in strain rate remains almost unaltered at a fixed strain value. So it is straightforward to argue that, during the transient change in strain rate, the possibility of generation of significant amount of mobile dislocations could be ruled out.

According to the Orowan equation (Kocks et al 1975), the plastic strain rate, $\dot{\varepsilon}$, is expressed in terms of mobile dislocation density, $\rho_{\mathrm{m}}$, and velocity, $v$, as

$$
\dot{\varepsilon}=\rho_{\mathrm{m}} b v,
$$

where $b$ is the magnitude of Burger's vector. As $\rho_{\mathrm{m}}$ is a constant, a sudden increase in the strain rate caused an increase in the dislocation velocity only.

The true stress, $\sigma$, at the true strain value of $11.33 \%$, increased for all the cases when the strain rate was changed. The variation of $\sigma$ with time was plotted (figure 3 ) and found to fit well with the Boltzmann type Sigmoid function of the form,

$$
\sigma(t)=\frac{\sigma_{i}-\sigma_{f}}{\left(1+e^{\frac{t-t_{0}}{\Delta t}}\right)}+\sigma_{f} .
$$

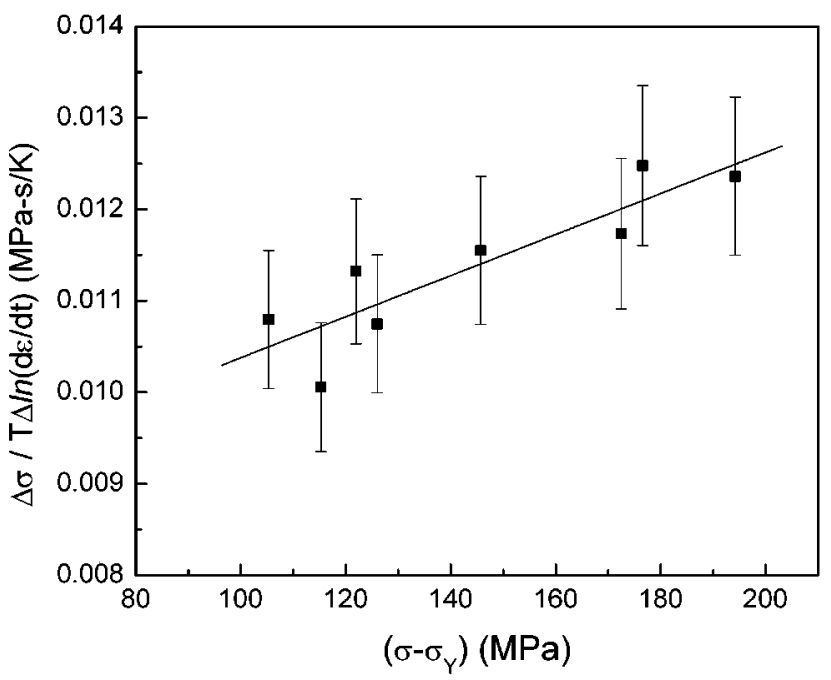

Figure 2. Haasen plot for the specimens at room temperature within an error of $\pm 5 \%$. 


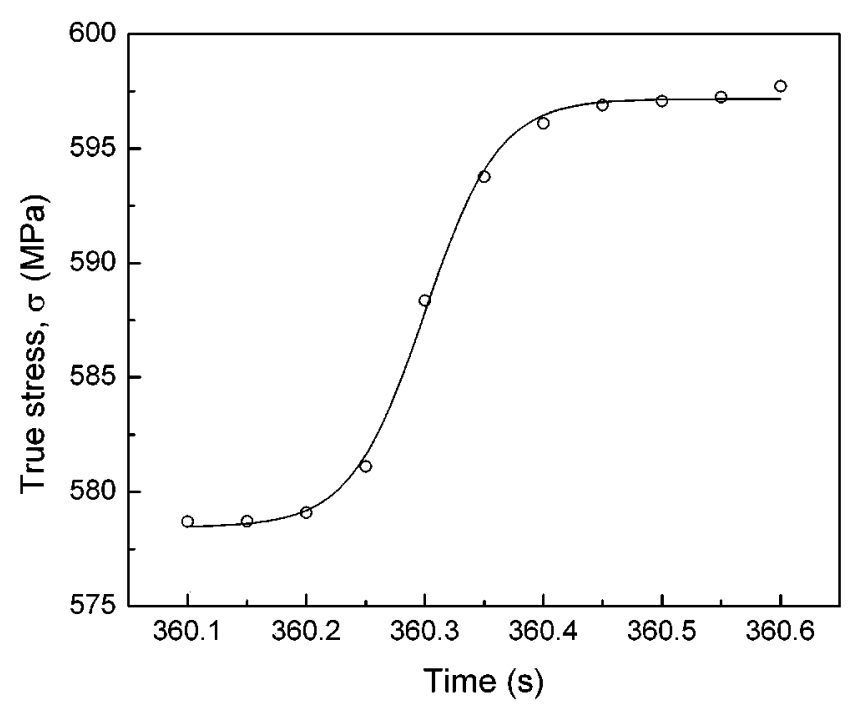

Figure 3. The experimental data of true stress with time (open circles) at the juncture of increase in final strain rate fitted with a Sigmoid function. The solid line is the fitted curve.

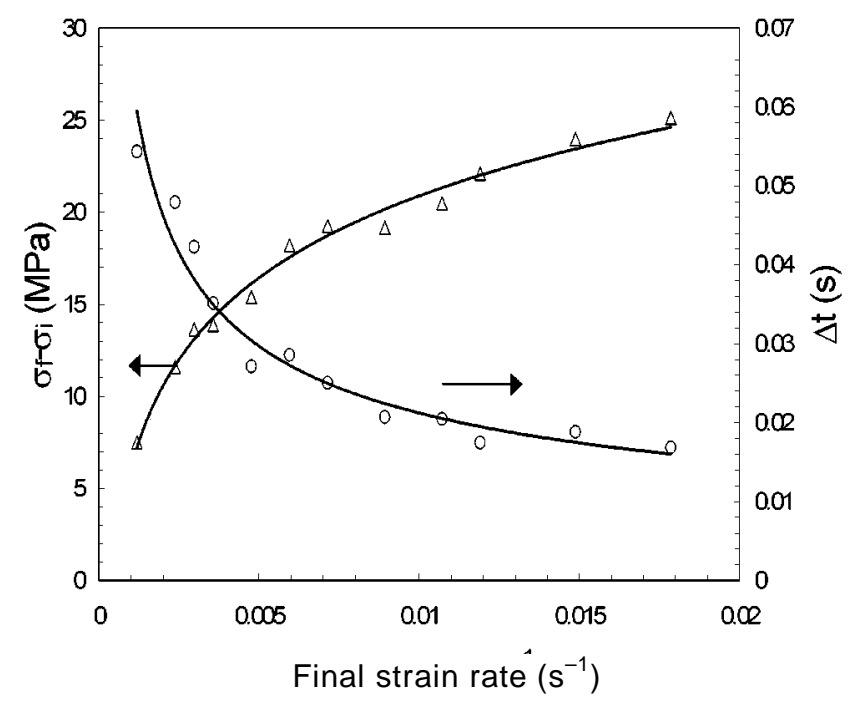

Figure 4. Variation of $\Delta t$ and $\left(\sigma_{f}-\sigma_{i}\right)$ with final strain rate values.

Here, $\sigma_{i}$ and $\sigma_{f}$ are the stress values for $t\left\langle t_{0}\right.$ and $\left.t\right\rangle t_{0}$, respectively, $t_{0}$ being the time at which $\sigma\left(t_{0}\right)=\left(\sigma_{i}+\sigma_{f}\right) / 2$ and $\Delta t$ is related to the width of the Sigmoid. The best fitted values of the parameters, $\Delta t$ and $\left(\sigma_{f}-\sigma_{i}\right)$ for different final strain rates $\left(\dot{\varepsilon}_{2}\right)$ are shown in figure 4 . From figure 4 , it is interesting to note that the increment in stress, $\left(\sigma_{f}-\sigma_{i}\right)$, increased and $\Delta t$ decreased with $\dot{\varepsilon}_{2}$. Both of them showed asymptotic type of saturation at higher $\dot{\varepsilon}_{2}$.

The strain rate dependence of $\left(\sigma_{f}-\sigma_{i}\right)$ can be explained as follows. Using (5) and assuming the dislocation velocity, $v=A \sigma^{m^{\prime}}$ (A is a constant) (Dieter 1986), we obtained

$$
\ln \dot{\varepsilon}=\ln \left(A \rho_{m} b\right)+m^{\prime} \ln \sigma .
$$

As $\rho_{m}$ remained constant during transient change in strain rate, it can be shown that

$$
\sigma_{f}-\sigma_{i}=<\sigma_{i}>\left(\frac{\dot{\varepsilon}_{2}}{\dot{\varepsilon}_{1}}\right)^{1 / m^{\prime}}-<\sigma_{i}>,
$$

where $\left\langle\sigma_{i}\right\rangle$ is the average initial true stress. Using $\left\langle\sigma_{i}\right\rangle=$ $561 \cdot 16 \mathrm{MPa}$ for all the tested samples and fitting (8) with the data shown in figure 4, we obtained $m^{\prime}=97.08$ which lead to (Dieter 1986) $m=1 / m^{\prime}=0 \cdot 0103$. This provided an excellent match with the average strain rate sensitivity of $0 \cdot 01$ obtained from the experiments. The $\Delta t$ followed a power law behaviour with final strain rate of the form,

$$
\Delta t=\Delta t_{0}+C\left(\dot{\varepsilon}_{2}\right)^{\alpha}
$$

where $\Delta t_{0}=12.2 \times 10^{-3} \mathrm{~s}, C=0.0004$ and $\alpha=-0 \cdot 7$. This phenomenological power law dependence was only possible as the velocity of dislocations saturates with increasing stress field.

\section{Conclusions}

The stress-strain measurements were carried out in the low-carbon steel specimen at room temperature following a sudden strain rate change in its plastic deformation regime. The strain rate sensitivities were calculated for different final strain rates. It was found from the analysis of Haasen plot that the transient change in the strain rate in plastic regime was associated with a change in the average dislocation velocity, instead of a change in the dislocation density.

\section{References}

Dieter G E 1986 Mechanical metallurgy (New York: McGraw Hill) 3rd ed

Haasen P 1958 Philos. Mag. 3384

Hall E O 1951 Proc. Phys. Soc. London 643747

Hayakawa $\mathrm{H}$ et al 2003 Jap. Inst. Metals 6722

Hirth J P and Lothe J 1982 Theory of dislocations (New York: Wiley) 2nd ed.

Kaschner G C and Gibeling J C 1996 Scr. Mater. 351397

Kocks U F, Argon A S and Ashby M F 1975 Prog. Mater. Sci. 191

Kok S et al 2003 Acta Mater. 513651 and references therein Mignogna R et al 1970 Met. Trans. 11771

Mulford R A 1979 Acta Metal. 271115

Nabarro F R N 1979-1987 Dislocation in solids (Amsterdam: North Holland) Vols 1-7

Onodera R J 2002 Jap. Inst. Metals 6660

Peierls R E 1940 Proc. Phys. Soc. London 5234

Petch N J 1953 J. Iron Steel Inst. London 17325

Reid N C et al 1965 Philos. Mag. 12409

Suzuki T et al 1991 Dislocation dynamics and plasticity (Berlin: Springer-Verlag Berlin and Heidelberg GmbH \& Co.) 